ORIGINAL ARTICLE

AFRICAN JOURNAL OF CLINICAL AND EXPERIMENTAL MICROBIOLOGY SEPTEMBER 2013 ISBN 1595-689X VOL14 No.3

AJCEM/1322

COPYRIGHT 2013

http://www.ajol.info/journals/ajcem

AFR. J. CLN. EXPER. MICROBIOL. 14(3): 164-168. http:/ / dx.doi.org/10.4314/ajcem.v14i3.8

\title{
MULTIPLE ANTIBIOTIC RESISTANCE (MAR) INDICES OF PSEUDOMONAS AND KLEBSIELLA SPECIES ISOLATES IN LAGOS UNIVERSITY TEACHING HOSPITAL
}

\author{
Osundiya $\mathrm{OO}^{1}$, Oladele $\mathrm{RO},{ }^{2}$ Oduyebo $\mathrm{OO}^{2}$ \\ 1Department of Biological Sciences, Redeemer's University, Ogun state. Nigeria \& ${ }^{2}$ Department of Medical Microbiology \\ \& Parasitology, College of Medicine of the University of Lagos, P.O. Box 12003, Lagos, Nigeria. \\ Correspondence: Dr. R.O.OLADELE, Dept of Med Micro \& Parasitology, College of Medicine, University of Lagos, Lagos, \\ Nigeria. Email -drritaoladele@yahoo.com; roladele@cmul.edu.ng
}

\begin{abstract}
ABSRACT
Background/Objectives: Pseudomonas and Klebsiella infections are important nosocomial infections because of the attendant significant morbidity, mortality and socio-economic impact. These infections are difficult to treat due to the innate and acquired resistance mediated by the organisms' genome and other transferable genetic elements. We determined the multiple antibiotic resistance indices of Pseudomonas spp and Klebsiella spp isolated from clinical specimens in Lagos University Teaching Hospital.

Methods: 110 clinical isolates were evaluated using Microbact ${ }^{\mathrm{TM}}$ 24E (Oxoid, UK) and Pseudomonas and Klebsiella species isolates were 34 and 21 respectively. The antimicrobial susceptibility patterns of the Pseudomonas and Klebsiella isolates were determined by Kirby-Bauer's disc diffusion method and results interpreted by CLSI interpretative values. Multiple Antibiotic Resistance index (MAR) were calculated.

Results: MAR index of the Pseudomonas and Klebsiella samples was 0.4, as 31 (91.2\%) and 14 (66.7\%) of Pseudomonas spp and Klebsiella spp respectively were multi drug resistant. Isolates of Pseudomonas spp demonstrated the highest level of resistance to Ceftazidime (79.4\%), Cefixime (76.5\%), Cedipime (50\%) and Piperacillin $(\mathbf{4 4 . 1 \% )}$; while that of Klebsiella spp were carbenicillin $(76.4 \%)$, pipericillin $(71.4 \%)$, cefixime $(52.4 \%)$ and cefradoxil $(42.9 \%)$ respectively. There was a low level of resistance to quinolones and aminoglycosides.

Conclusion: The MAR index shows increase in the rates of resistance among these organisms thus making antimicrobial susceptibility surveillance and testing more crucial in selecting empiric regimen or definitive treatment.
\end{abstract}

KEY WORDS: Lagos, multiple antibiotic resistance (MAR), antimicrobial susceptibility, Pseudomonas and Klebsiella spp.

\section{INTRODUCTION:}

The World Health Organization (WHO) estimated that infections accounted for $45 \%$ of deaths in Africa and South-East Asia and were responsible for $48 \%$ of premature deaths worldwide, bacterial infection accounted for a significant proportion of these infections in Africa [1]. The valuable life spans of most antibiotics used in treating these infections have been diminished by the development of resistant strains. Microorganisms use a combination of mechanisms in developing resistance however a dominant mechanism may be identifiable [2,3]. The relationship between antibiotic use and resistance is complex; a major driving factor for antibiotic resistance is antibiotic use/abuse both within medicine and veterinary medicine. In Nigeria 53\% of respondents in a survey took incomplete regimen of antibiotics, a significant proportion of which were self prescribed for unspecified ailments $[4,5]$.

Antibiotic resistance increasingly compromises the outcome of many infections that were, until recently, treatable and remain the most common diseases in Africa [6]. The global problem of antimicrobial resistance is particularly pressing in developing countries, where the infectious disease burden is high and cost constraints prevent the widespread application of newer, more expensive agents [6]. Reports from multiple studies from

different parts of Nigeria though have observed temporal trends in the prevalence of resistance among enteric organisms have shown increasing prevalence in the last fifteen years [7].

High prevalence of multidrug resistance indicates a serious need for broad-based, local antimicrobial resistance surveillance and planning of effective interventions to reduce multidrug resistance in such pathogens [8]. Multiple antibiotic resistance (MAR) in bacteria is most commonly associated with the presence of plasmids which contain one or more resistance genes, each encoding a single antibiotic resistance phenotype [9].

Emergence of antibiotic resistance in $P$. aeruginosa resulting in severe adverse outcomes had been on the rise. In the USA annual prevalence of fluoroquinolones and imipenem resistant $P$. aeruginosa increased from $15 \%$ in 1991 to $41 \%$ in $20001 \%$, and from 1989 to 2006 from $13 \%$ to $20 \%$ respectively [10]. In Lagos, similar results have been previously documented [11]. These same conclusions had been drawn in Zaria though $P$. aeruginosa there were more susceptible to 
fluoroquinolones [9]. In Zaria and Enugu about 60 $\%, 42 \%, 33 \%$ of clinical isolates of Pseudomonas spp, Escherichia spp, and Klebsiella spp respectively were noted to be multidrug resistant [12]. In Enugu and Abakaliki $62 \%$ of Klebsiella isolates were ESBL producers.

Multiple antibiotic resistance (MAR) indexing has been shown to be a cost effective and valid method of bacteria source tracking. Multiple antibiotic resistance index is calculated as the ratio of number of resistant antibiotics to which organism is resistant to total number of antibiotics to which organism is exposed [13, 14]. MAR index values greater than 0.2 indicate high risk source of contamination where antibiotics are often used.

This study was carried out to evaluate the MAR indexes values of clinical isolates of Pseudomonas spp and Klebseilla spp from Lagos University Teaching Hospital (LUTH).

\section{METHOD}

Pre-identified clinical isolates of gram negative bacilli species (110 in number) were obtained from Lagos University Teaching Hospital (LUTH) Microbiology laboratory between June and September 2010 and preserved on nutrient agar for further identification. Isolates sub-cultured from nutrient agar slants onto MacConkey agar medium were identified by a combination of colonial morphological characteristics, gram stain, and motility. Further biochemical characterisation was done using Microbact ${ }^{\mathrm{TM}}$ 24E (Oxoid, UK). Antimicrobial susceptibility pattern of the isolates were done after they were re-identified. Antimicrobial susceptibility testing was performed using the disc diffusion method (Bauer et al 1966). Antibiotic discs tested were Cefixime, Cedipime, Cefradoxil, Ceftazidime, Cefuroxime, Gentamycin, Amikacin, Piperacillin, Carbenicillin, Ciprofloxacin, Ofloxacin, Imipenem (Oxoid, UK)

Values obtained were interpreted according to the Clinical and Laboratory standards Institute (CLSI, 2010) into resistant, intermediate and sensitive categories. Apart from general caution to strictly follow procedures as described in manufacturer's manual, Pseudomonas aeruginosa ATCC 2785 and Escherichia coli ATCC 25922 were used as positive and negative controls respectively.

MAR index was determined by following the procedure described by Krumperman 1983 [14]. A MAR index for an isolate is calculated as: Number of antibiotics to which isolate is resistant/ Total number of antibiotics against which isolate was tested.

\section{RESULTS}

Total no of clinical isolates obtained from LUTH laboratory was 110 . Morphological and biochemical identification revealed that $34(30.9 \%)$ of these were Pseudomonas species while 21(19.1\%) and $22(20 \%)$ were Klebsiella and Escherichia species. The rest $33(30 \%)$ were made up by a diverse group of the family Enterobacteriacae namely Serratia, Acinetobacter, Enterobacter, Acetobacter and Citrobacter species.

Overall antibiotics prescribed by physicians working in all units in LUTH in order of descending frequency included Ampicillin/Cloxacillin, Amoxicillin/Clavulanic acid, Gentamycin, Ciprofloxacin, Cefuroxime, Ceftazidime, Ceftriaxone, Pefloxacin, Ofloxacin, Imipenem.

The total proportion of isolates found sensitive to all antibiotics used in antimicrobial susceptibility testing in Pseudomonas and Klebsiella spp were 58.4\% and $52.2 \%$ respectively.

Table 1 and figure 1 show the resistance pattern of Pseudomonas to each antibiotic used in the antimicrobial susceptibility testing. Ceftazidime 27 $(79.4 \%)$ demonstrated the highest levels of resistance followed by Cefixime $26(76.5 \%)$ while other cephalosporins demonstrated moderate activity against Pseudomonas. Isolates were most sensitive to Imipenem 32 (94.15), Genticin 31 (91.2\%), and Ciprofloxacin 29 (85.3\%). $76.5 \%$ of isolates were sensitive to Ofloxacin and Amikacin. Nearly all isolates 31(91.2\%) had Multiple Antibiotic Resistance (MAR) index that was higher 0.2 only $3(8.8 \%)$ had a MAR of 0.2 or less. MAR index of the Pseudomonas sample is 0.4 .

Table 2 shows the resistance pattern of Klebsiella to each antibiotic used in the antimicrobial susceptibility testing. The highest levels of resistance were seen in Piperacillin and Ceftazidime 15 (71.4\%); other cephalosporin's also demonstrated high levels of resistance. Isolates were most sensitive to Imipenem $20(95.2 \%)$ followed by Gentamycin 19 (90.5\%), Ciprofloxacin 19 (90.5\%) and Cedipime $18(85.7 \%)$ respectively. Ofloxacin and Amikacin were moderately active against Klebsiella isolates. Only a third of the isolates were resistant to two or less antibiotics. The proportion of isolates with Multiple Antibiotic Resistance (MAR) index greater than 0.2 was $66.7 \%$ (14) while those with MAR index less than 0.2 was $33.3 \%$ (7) showing that a greater proportion of the isolates are likely to be from a high risk source. The MAR index of the Klebsiella sample was 0.4.

Figures 1 \&2 demonstrate the comparative sensitivity pattern of Pseudomonas and Klebsiella isolates to selected antibiotics in use in the hospital. 
TABLE 1: ANTIMICROBIAL SUSCEPTIBILITY TESTING BY DISC DIFFUSION METHOD OF PSEUDOMONAS ISOLATES

\begin{tabular}{|c|c|c|c|c|c|}
\hline \multicolumn{6}{|c|}{ Susceptibility Rates } \\
\hline Antibacterial Agent & & $\mathbf{n}$ & $\% \mathrm{~S}$ & $\% \mathbf{I}$ & $\% \mathbf{R}$ \\
\hline Cefixime & CFM & 34 & 17.7 & 5.9 & 76.5 \\
\hline Cedipime & FEP & 34 & 47.1 & 2.9 & 50 \\
\hline Cefradoxil & CFR & 34 & 50 & 2.9 & 47.1 \\
\hline Ceftazidime & $\mathrm{CA}_{2}$ & 34 & 17.7 & 2.9 & 79.4 \\
\hline Cefuroxime & CXM & 34 & 85.3 & 2.9 & 11.8 \\
\hline Imipenem & IPM & 34 & 94.1 & 0 & 5.9 \\
\hline Piperacillin & PRL & 34 & 55.9 & 0 & 44.1 \\
\hline Carbenicillin & CAR & 34 & 58.8 & 5.9 & 35.3 \\
\hline Ciprofloxacin & CIP & 34 & 85.3 & 0 & 14.7 \\
\hline Ofloxacin & OFX & 34 & 76.5 & 5.9 & 17.7 \\
\hline Gentamycin & GN & 34 & 91.2 & 2.9 & 5.9 \\
\hline Amikacin & AK & 34 & 76.5 & 8.8 & 14.7 \\
\hline
\end{tabular}

$\mathrm{n}=$ no of isolates, $\mathrm{S}=$ sensitive, $\mathrm{I}$ - intermediate, $\mathrm{R}=$ resistant (according to CLSI

TABLE 2: ANTIMICROBIAL SUSCEPTIBILITY TESTING BY DISC DIFFUSION METHOD FOR KLEBSIELLA ISOLATES

\begin{tabular}{|c|c|c|c|c|c|}
\hline \multirow[b]{2}{*}{ Antibacterial Agent } & & \multicolumn{4}{|c|}{ Susceptibility Rates } \\
\hline & & $\mathbf{n}$ & $\% \mathrm{~S}$ & $\% \mathbf{I}$ & $\% \mathbf{R}$ \\
\hline Cefixime & CFM & 21 & 38.1 & 4.8 & 52.4 \\
\hline Cedipime & FEP & 21 & 85.7 & 4.8 & 9.5 \\
\hline Cefradoxil & CFR & 21 & 38.1 & 19 & 42.9 \\
\hline Ceftazidime & $\mathbf{C A}_{2}$ & 21 & 61.9 & 0 & 38.1 \\
\hline Cefuroxime & CXM & 21 & 47.6 & 28.6 & 23.8 \\
\hline Imipenem & IPM & 21 & 95.2 & 0 & 4.8 \\
\hline Piperacillin & PRL & 21 & 28.6 & 0 & 71.4 \\
\hline Carbenicillin & CAR & 21 & 9.5 & 48 & 76.4 \\
\hline Ciprofloxacin & CIP & 21 & 90.5 & 0 & 9.5 \\
\hline Ofloxacin & OFX & 21 & 61.9 & 0 & 38.1 \\
\hline Gentamycin & GN & 21 & 90.5 & 0 & 9.5 \\
\hline Amikacin & AK & 21 & 57.1 & 14.3 & 28.6 \\
\hline
\end{tabular}

$\mathrm{n}=$ no of isolates, $\mathrm{S}=$ sensitive, $\mathrm{I}$ - intermediate, $\mathrm{R}=$ resistant (according to CLSI 


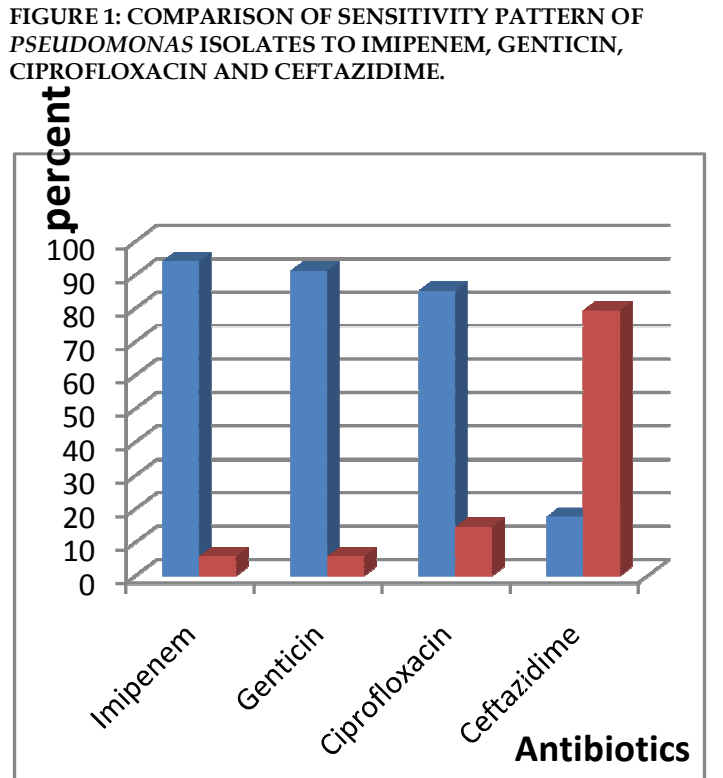

Blue bars represent proportion that is sensitive;

Red bars represent proportion that is resistant.

\section{DISCUSSION}

High prevalence of multidrug resistance indicates a serious need for antibiotics surveillance program. Multiple antibiotic resistance (MAR) analysis has been used to differentiate bacteria from different sources using antibiotics that are commonly used for human therapy. Compared to other methods of bacteria source tracking such as genotypic characterization, the MAR indexing method is costeffective, rapid and easy to perform. It is also simple and does not require specialized training and expensive equipment [13]. The monitoring of both antibiotic consumption and multiple antibiotic resistances (MAR) especially in nosocomial infections is critically necessary to setting up of effective containment programs and audit of such programs [13, 15].

Pseudomonas and Klebsiella are major pathogens in healthcare associated infections (HAI)[12]. This not only because of the attendant significant morbidity associated with infections but also because increasing rates of resistance in them is making it more difficult for them to be treated with cheaper first line antibiotics. Emerging and increasing resistance to newer and otherwise efficacious antibiotics may compound the whole problem[1]. The $30.9 \%$ and over $19 \%$ prevalence obtained for pseudomonas and klebsiella respectively out of a sample of 110 clinical isolates approximate to prevalence previously obtained in centre of study $[11,16]$
FIGURE 2: COMPARISON OF SENSITIVITY PATTERN OF KLEBSIELLA ISOLATES TO IMIPENEM, GENTICIN, CIPROFLOXACIN AND CEFTAZIDIME.

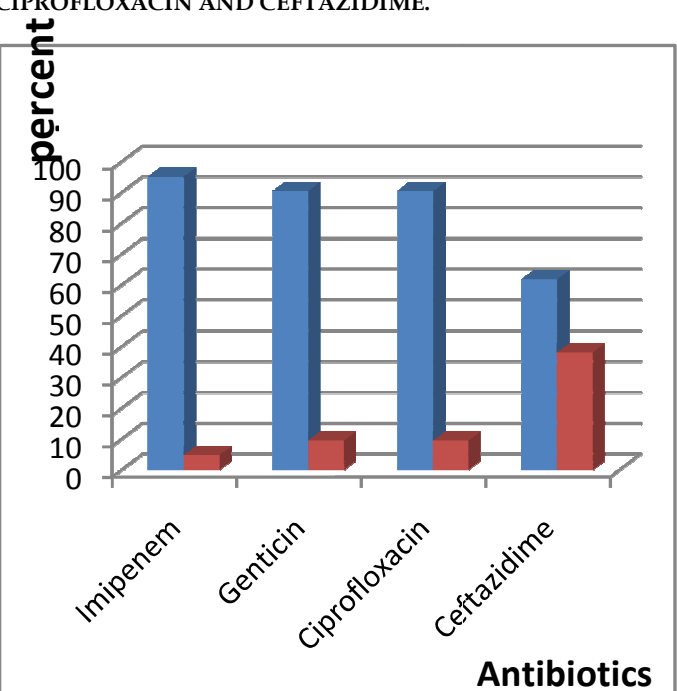

Blue bars represent proportion that is sensitive; Red bars represent proportion that is resistant.
Isolates of both genera demonstrated high levels of susceptibility to the first three drugs (imipenem 32 (94.15\%), genticin $19(91.2 \%)$ and ciprofloxacin 29 (85.3\%) for pseudomonas and imipenem 20 (95.2\%), Genticin 19 (90.5\%), Ciprofloxacin 19 (90.5\%) for klebsiella spp while varying levels of resistance from moderate to high levels resistance were demonstrated to the cephalosporins. This finding is consistent with that of other studies which reported low resistance profiles for the quinolones and aminoglycosides respectively[8, 12, 16]. However the difference in resistance rates in these works may be (in spite of general similar low rates profile for these drugs) explained by the increasing trend that had been noted globally [1]. This may have implications on the effectiveness of the local and probably national hospital infection and antibiotic resistance control programs. The moderate to high levels of resistance in the cephalosporins underscore the emergence of B-lactamases, specifically cephalosporinases among resistant strains of these organisms as previously reported $[2,6,10.14 .15]$. This compromises the clinical efficacy of a very important class of drugs commonly used in the management of many infections in this environment. The clearly visible high rate of resistance of isolates to penicillin (Piperacillin, Carbenicillin) corroborates documented increasing penicillinase-producing B-lactamases strains among these organisms [10].

Analysis of the MAR index of isolates showed that $91.2 \%$ and $66.7 \%$ of Pseudomonas and Klebsiella 
isolates were resistant to three or more antibiotics. These figures were higher than was seen in the series done by Olayinka et al. This may be due to either the difference in the definition used for multidrug resistance in both works or may be an indication of a significant jump in the emergence of multi-drug resistance strains. Olayinka et al in their series estimated their proportion on the basis of resistance to four drugs or more. Three was used in this study as it approximates more with the general definition of multi-drug resistance [2].

\section{REFERENCES}

1) Okeke, I.N. and Sosa, A. Antibiotic resistance in Africa - Discerning the enemy and plotting a defence. Afr Health 2003.25(3); 10-15.29

2) Lambert, P.A. Mechanisms of antibiotic resistance in Pseudomonas aeruginosa. $J R$ Soc Med 2002; 95 ( 41):22-26.

3) Schweizer H P. Efflux as a mechanism of resistance to antimicrobials in Pseudomonas aeruginosa and related bacteria: unanswered questions. Genet Mol. Res 2003. 2(1): 48-62

4) Yah, S. C., Eghafona, N. O., Enabulele, I. O., Aluyi, H. A. S. Ampicillin Usage and Ampicillin Resistant (Ampr) Plasmids Mediated Escherichia Coli Isolated from Diarrheagenic Patients Attending Some Teaching Hospitals in Nigeria. Shiraz EMed J 2006; 7.

5) Yah, S. C., Yussuf, O. E. and Eghafona, N. O. Pattern of antibiotic usage by adult populations in the city of Benin, Nigeria. Scientif Res Essay 2008; 3: 081-085.

6) Okeke IN, Laxminarayan R., Bhutta ZA et al. Antimicrobial resistance in developing countries. Part 1: recent trends and current status. The Lancet Inf. Dis. 2005. 5:8; 481493.

7) Okeke, I.N., Fayinka S.T. and Lamikanra A. Antibiotic resistance trends in Escherichia coli from apparently healthy Nigerian students (1986-1998). Emerg Infect Dis; 2000; 6:393- 396.

8) Olayinka, B.O., Olonitola, O.S. and Olayinka, A.T. and Agada, E. A. Antibiotic susceptibility pattern and multiple antibiotic resistance index of Pseudomonas aeruginosa urine isolates from a University Teaching Hospital. Afr J Clin Exp Microbiol 2004; 5 (2): 198-202.

9) Daini, O. A., Ogbolu, O. D. and Ogunledun, A. (2005). Quinolone
All these findings have implications for the choice of antibiotics for empiric management of infections, continuous surveillance of antibiotic susceptibility patters and effective hospital infection control. Bacterial strains resistant to most classes of antibiotics will continue to emerge unless inappropriate uses of drugs are curtailed and continuous education of infection control practices maintained.

resistance and R-plasmids of some Gram negative enteric bacilli. Afr J Clin Exper Miccrobiol 2005; 6 (1): 14-20.

10) Lautenbach, E., Weiner, M.G., Bilker, W.B., Vo, L., Synnestedvet, M., Schein, J. and Kim, M. Imipenem Resistance in Pseudomonas aeruginosa: Emergence, Epidemiology, and Impact on Clinical and Economic Outcomes. Infect Cont Hosp Epidem 2010; 31:47-53.

11) Aibinu, I., Nwanneka, T. and Odugbemi, T. Occurrence of ESBL and MBL in Clinical Isolates of Pseudomonas aeruginosa From Lagos, Nigeria. J Amer Sci 2007; 3(4): 81-85.

12) Olayinka, A.T., Onile, B.A. and Olayinka, B.O. Antibiotic susceptibility and plasmid pattern of Pseudomonas aeruginosa from the surgical unit of a university teaching hospital in north central Nigeria. Int J Med and Med Sci 2009; 1(3): 079-083.

13) Mthembu, M.S. The usefulness of multiple antibiotic resistance (MAR) indexing technique in differentiating faecal coliform bacteria from different sources. Thesis (Msc) University of Zululand; 2008.

14) Krumperman, P.H., $1983 . \quad$ Multiple antibiotic resistance indexing of Escherichia coli to indentify high-risk sources of fecal contamination of foods. Applied Environ. Microbiol 1983; 46: 165-170.

15) Kamat, U.S., Ferreira, A.M., Savio, R. and Motghare, D.D. Antimicrobial Resistance Among Nosocomial Isolates in a Teaching Hospital in Goa. Ind J Comm Med 2008; 33 (2): 89-92.

16) Oduyebo OO, Ogunsola FT, Odugbemi T. Prevalence of multi-resistant strains of P.aeruginosa isolated at the Lagos University Teaching Hospital from 19941996. Nig Quart J Med 1997; 7: 373-376. 